\title{
The Anti-Microbial Peptide LL-37/CRAMP Is Elevated in Patients with Liver Diseases and Acts as a Protective Factor during Mouse Liver Injury
}

\author{
Svenja Wertenbruch ${ }^{a}$ Hannah Drescher ${ }^{a}$ Vera Grossarth ${ }^{a}$ Daniela Kroy ${ }^{a}$ \\ Arne Giebeler $^{b}$ Stephanie Erschfeld ${ }^{a}$ Daniel Heinrichs ${ }^{c}$ Oliver Soehnlein ${ }^{e}$ \\ Christian Trautwein $^{a} \quad$ Lars-Ove Brandenburg ${ }^{d}$ Konrad Streetz ${ }^{a}$ \\ ${ }^{a}$ Department of Internal Medicine III, University Hospital RWTH Aachen, ${ }^{b}$ Department of Surgery, University \\ Hospital RWTH Aachen, 'Institute of Biochemistry and Molecular Cell Biology, University Hospital RWTH Aachen, \\ and ${ }^{\mathrm{d}}$ Institute of Anatomy, University Hospital RWTH Aachen, Aachen, and ${ }^{\mathrm{e}}$ Institute for Cardiovascular Prevention, \\ LMU Munich, Munich, Germany
}

\section{Key Words}

Antimicrobial peptides · LL-37 · CRAMP · Bile-duct-ligation

\begin{abstract}
Background: Antimicrobial peptides (AMP) are an important defense mechanism of the innate immune system and can modulate the course of various diseases. However, their significance during liver pathogenesis is currently not well defined. Methods: Patients with liver diseases were analyzed for LL-37/CRAMP, human beta-defensin-2 (hBD2), and complement $5 a$ (C5a) serum levels. Mice deficient in CRAMP (Cathelicidin-related Antimicrobial Peptide), the mouse homolog for human LL-37, were fed with a methionine- and choline-deficient diet (MCD) and underwent bile-duct ligation (BDL). Results: First, serum samples from patients with chronic liver diseases were investigated. Therefore, significantly enhanced levels for LL-37, hBD2, and complement C5a were detected, all of which comprise antimicrobial properties. Next, CRAMP-knockout (CRAMP-KO) mice were investigated, to better define a functional role of LL-37/ CRAMP in animal models of liver diseases. MCD feeding and bile-duct ligation of CRAMP-KO mice resulted in an enhanced degree of liver injury during the early treatment
\end{abstract}

phase. MCD feeding in CRAMP-KO mice led to stronger intrahepatic fat accumulation and significantly enhanced matrix remodeling, whereas BDL caused more extensive liver necrosis. At the late 28 days time point, MCD-fed CRAMP-KO mice displayed a higher intrahepatic fat load. Long-term changes in bile-duct-ligated mice included higher collagen content as a sign of enhanced fibrosis progression if CRAMP was absent. Conclusion: The study shows a clear correlation of antimicrobial peptide serum levels in patients with chronic liver diseases. Furthermore, we were able to demonstrate protective functions of LL-37/CRAMP in two independent mouse models of chronic liver injury. @ $2015 \mathrm{~S}$. Karger AG, Basel

\section{Introduction}

The innate immune system plays a crucial role during the defense against microbes as well as in the initiation of the inflammatory response. Here, antimicrobial peptides (AMPs) serve as an evolutionary well-conserved and im-

S.W., H.D., L.-O.B. and K.L.S. contributed equally to this publication.

\begin{tabular}{llll}
\hline KARGER 125/\% & ( 2015 S. Karger AG, Basel & & Konrad Streetz \\
& $0012-2823 / 15 / 0914-0307 \$ 39.50 / 0$ & & Department of Internal Medicine III, University Hospital RWTH Aachen \\
& & Pauwelstrasse 30 \\
E-Mail karger@karger.com & & DE-52074 Aachen (Germany) \\
www.karger.com/dig & & E-Mail kstreetz@ ukaachen.de
\end{tabular}


portant defense mechanism against bacteria and fungi in eukaryotic organisms. AMPs are usually produced and secreted by epithelial cells and lymphocytes, mainly granulocytes [1].

LL-37, the human homolog of the cathelicidin-related antimicrobial peptide (CRAMP), is the sole member of the human cathelicidin family. Its biologic function is primarily the initiation of bacterial lysis through the formation of wall pores in prokaryotes. Besides its direct antimicrobial function, it also has so far not yet thoroughly defined immune modulatory functions. The peptide comprises both pro- and anti-inflammatory properties that are modulated by the tissue environment and specific diseases [2]. Its proinflammatory phenotype becomes evident during monocyte differentiation, where it can drive cell plasticity toward M1 macrophage differentiation and IL-10 downregulation [3]. There is also a strong anti-inflammatory function through a modulation of TLR signaling described [4]. By downregulating the LPS-driven TLR4 response, it can thus prevent macrophage activation and the production of proinflammatory cytokines [5]. Its expression is regulated through inflammatory signaling [6], the vitamin-D pathway [7], and endoplasmatic reticulum stress [8]. Previous experiments using mice deficient in the murine ortholog CRAMP have demonstrated the importance of this peptide during the antimicrobial defense and inflammation $[9,10]$. Thus, it was shown that LL-37/CRAMP is required to prevent bacterial infections in the skin, the colon, and the urinary tract. Moreover, we have recently demonstrated further antimicrobial functions of the peptide in rodent models of meningitis and cerebral infection $[11,12]$.

With regard to the liver, it is known that activation of toll-like receptor (TLR) signaling - mostly through pathogen-associated molecular pattern molecules (PAMP) - is one of the major inflammatory pathways that promote progression of chronic hepatic diseases [13]. This is evident for both metabolic and cholestatic diseases, which ultimately result in the development of fibrosis and organ insufficiency. Here, bacterial toxins from the gut sustain an inflammatory response, which contributes to hepatic kupffer cell activation, promotes stellate cells to myofibroblast differentiation and the subsequent production of extracellular matrix as the morphologic correlate of fibrosis. Experiments in TLR4-deficient mice demonstrated that targeted disruption of this signaling pathway can efficiently prevent the development of hepatic fibrosis in the cholestatic mouse model of bile-duct ligation [14].

Within this study, we first aimed at correlating AMP serum level with the presence of liver diseases. This might be helpful to better define patients' prognosis or response to therapies. To more thoroughly define a functional role for AMPs during the course of liver diseases, as suggested in our own previous study [15], we applied CRAMP-deficient $(\mathrm{KO})$ mice to respective animal models. Especially models of metabolic and cholestatic injury are of interest, as here relevance for an involvement of AMPs due to a disturbed gut-liver axis is likely.

\section{Materials and Methods}

\section{Ethical Statement}

This study was carried out in strict accordance with the recommendations of the Ethics of the regional authorities for nature, environmental and consumer protection of North RhineWestfalia (LANUV - Landesamt für Natur, Umwelt und Verbraucherschutz NRW) Recklinghausen, Germany, and approved by the LANUV Committee (Permit Number: TV10695G1). All surgeries were performed under ketamin-hydrochloride/xylazin-hydrochloride anesthesia, and all efforts were made to minimize suffering.

\section{Housing and Generation of Mice}

Both CRAMP knockout [16] and the corresponding wildtype (WT) mice were used as experimental animals in a C57BL/6 background. At least five mice per group were treated and analyzed in parallel for all experiments, which were repeated at least twice. Mice were housed in $12 \mathrm{~h}$ light/dark cycles with water and food freely available in the animal facility of the University Hospital RWTH Aachen, and were treated in accordance with the criteria of the German administrative panel on laboratory animal care.

\section{Bile-Duct Ligation/MCD Treatment}

Eight- to ten-week-old mice were subjected to bile-duct ligation (BDL) as indicated. BDL was performed in anesthetized mice by tying the common bile duct using a non-absorbable filament (Ethicon, Boston, Mass., USA). For each of the following analysis, at least 5 mice per genotype and time point were investigated. All experiments were performed at least twice.

For dietary treatment, 8-12-week-old male mice weighting $25 \mathrm{~g}$ were fed either a methionine- and choline-deficient diet (MCD) or a chow diet (MP Biomedicals, LLC, Ohio, USA). Both knockout and wild-type animals showed a food intake of $56 \mathrm{~g}$ per day without differences between chow and dietary treatment.

\section{Blood Collection}

For retro-orbital bleeding, mice were briefly anesthetized with isoflurane and blood was collected via a glass capillary. Samples were centrifuged at $10,000 \mathrm{rpm}$ for $10 \mathrm{~min}$, aliquoted and serum was stored at $-80^{\circ} \mathrm{C}$ until further analysis.

\section{Histology, Sirius-Red, and Oil-Red Staining}

Liver samples were either snap frozen in OCT compound or fixed in $4 \%$ formaldehyde, embedded in paraffin, cut, and stained with hematoxylin and eosin. Pictures were taken using an AxioImager Z1 (Carl Zeiss, Jena, Germany) for each time point per 
genotype. For Sirius-red staining, the paraffin sections were incubated in the Sirius-red-staining solution (Polysciences Inc., 55780, Eppelheim, Germany) for $1 \mathrm{~h}$. Thereafter, the slides were incubated in $0.1 \mathrm{M} \mathrm{HCl}$ for $5 \mathrm{~min}$, treated with an ascending ethanol series, incubated in Roti-Histol (Roth, Karlsruhe, Germany), and finally covered with Roti-Histokit (Roth, Karlsruhe, Germany). The sections were analyzed under polarized light. Photomicrographs of Sirius-red positive areas taken in a $10 \times$ magnification were analyzed via color error measurement using the open source software ImageJ. Then, collagen filaments were morphometrically analyzed and used for the calculation of collagen per area field. For oil-red staining, frozen sections were fixed in formalin, washed in PBS, and then stained with Oil-red-O staining solution (Sigma, Steindorf, Germany). After rinsing with water, nuclei were stained with hematoxylin.

\section{Immunofluorescence Stainings}

Liver cryosections $(5 \mu \mathrm{m})$ were air-dried and fixed with icecold acetone. After rehydration in phosphate-buffered saline (PBS), the samples were treated with $2 \mathrm{~N} \mathrm{HCl}$ for $30 \mathrm{~min}$ and afterward neutralized with $0.1 \mathrm{M}$ sodium borate ( $\mathrm{pH}$ 8.0) for $9 \mathrm{~min}$. The samples were washed in PBS. Antibodies were incubated in $0.2 \%$ bovine serum albumin (BSA) and $10 \%$ goat serum in PBS (anti-BrdU: 1:40; Becton Dickinson, Heidelberg, Germany) for $1 \mathrm{~h}$ at $37^{\circ} \mathrm{C}$ or overnight at $4^{\circ} \mathrm{C}$, respectively. For the detection of collagen tissue, sections were fixed in $4 \%$ PFA (Roth, Karlsruhe, Germany) and blocked in 2\% BSA in PBS acid. Incubation of the first antibody was performed overnight at $4^{\circ} \mathrm{C}$ or $1 \mathrm{~h}$ at room temperature, respectively (anti-collagen type 1: 1:250; Biotrend, Cologne, Germany; anti-Ki67: 1:100 in 0.3\% Triton X-100, 5\% goat serum in PBS; Nova Castra Laboratories, Newcastle upon Tyne, UK). After washing in PBS, Alexa Fluor 488 and/or 594-conjugated secondary antibodies (Molecular Probes/Invitrogen, Karlsruhe, Germany) were used for immunofluorescence detection. The sections were analyzed using a fluorescence microscope (Zeiss, Jena, Germany).

\section{Patient Collection and Statistics}

Serum samples of 30 patients without liver disease and of 80 patients with liver disease were collected in the Department of Gastroenterology, RWTH University Hospital Aachen, Germany. The local ethics committee approved the study protocol. Written informed consent was obtained from the patient, his or her spouse, or the appointed legal guardian. Levels of antimicrobial peptides human beta-Defensin-2, C5a, and LL37 were determined by ELISA as described next. Significances were analyzed by unpaired Student's t test and are indicated by ${ }^{*}\left({ }^{*} \mathrm{p}<0.05\right)$.

ELISA

Patient blood samples were prepared according to the instructions of the manufacturer of the ELISA kits after blood withdrawal and stored at $-80^{\circ} \mathrm{C}$ until further analysis. Importantly, serum samples were centrifuged twice to ensure any contamination of neutrophils and blood cellular material, which lead to false positive AMP values. All samples were run in duplicate to ensure result consistency. If values differed $>15 \%$, the particular sample was re-analyzed.

For serum analysis, we used the following assays: LL37, human, ELISA (Cat.\# HK321-02, Fa: Hycultec GmbH, Beutelsbach, Germany), human beta defensin-2, (human BD-2, Cat. No. K0331208, Fa: Komabiotech, Yangpyeong-dong, Korea), and Hu- man Complement Component C5a Duo Set (Cat. No. DY2037, Fa: R\&D Systems, Wiesbaden-Nordenstadt, Germany).

After applying the substrate 3,3',5,5'-tetramethylbenzidine-dihydrochloride (Sigma-Aldrich, Steinheim, Germany) and termination of the substrate reaction with sulfuric acid, the absorbance was measured in a fluorescent plate reader at a wavelength of $450 \mathrm{~nm}$. The absorbance values were converted to $\mu \mathrm{g} / \mathrm{ml}$ by comparison with a standard curve according to the instructions of the manufacturer.

\section{TUNEL Assay}

Cryosections $(5 \mu \mathrm{m})$ were air-dried and fixed with $4 \%$ paraformaldehyde at room temperature. After washing with PBS, the slides were incubated for $10 \mathrm{~min}$ in $3 \% \mathrm{H}_{2} \mathrm{O}_{2}$ methanol followed by a 2 -min incubation in sodium citrate $(0.1 \%)$. After washing in PBS, the substrate mixture was applied according to the manufacturer's instructions (Roche, Mannheim, Germany).

\section{Quantification and Statistics}

All numerical results are expressed as mean \pm SEM and represent data from at least four animals per time point. Calculations via manual counting of positive cells were done with five to 10 high power fields/liver. All significant $\mathrm{p}$ values were measured by the Student's t test. A value of $\mathrm{p}<0.05$ was considered significant $\left(^{*} \mathrm{p}<\right.$ $\left.0.05,{ }^{* *} \mathrm{p}<0.01,{ }^{* * *} \mathrm{p}<0.001\right)$.

\section{Results}

\section{Antimicrobial Peptides Are Positively Correlated with Chronic Liver Disease}

In order to analyze the importance of antimicrobial peptides during the development of chronic liver disease, we determined patient serum levels of two different antimicrobial peptides (AMPs: human beta-Defensin 2 (hBD2) and LL-37). In addition, we analyzed serum levels of complement $5 \mathrm{a}(\mathrm{C} 5 \mathrm{a})$, an important activator of the complement cascade, being also involved in bacterial lyses, inflammation, and chemotaxis and thus closely related to the function of AMPs. Serum samples of $30 \mathrm{pa}-$ tients without liver disease were compared with serum samples of 80 patients with chronic liver disease. Our analysis showed that patients with existing chronic liver disease displayed significantly elevated serum levels of hBD2, C5a, and LL-37 (fig. 1a-c). A further analysis of patients with various underlying liver disease entities unraveled that LL-37 serum levels during ethanol-induced injury, HVC, NASH, and cryptogenic liver diseases were significantly different from control patients (determined by unpaired Student's t test). Statistical significance could not be shown for patients with $\mathrm{HBV}$ and $\mathrm{PBC} / \mathrm{PSC}$, although also within those groups LL-37 serum levels were clearly increased. 


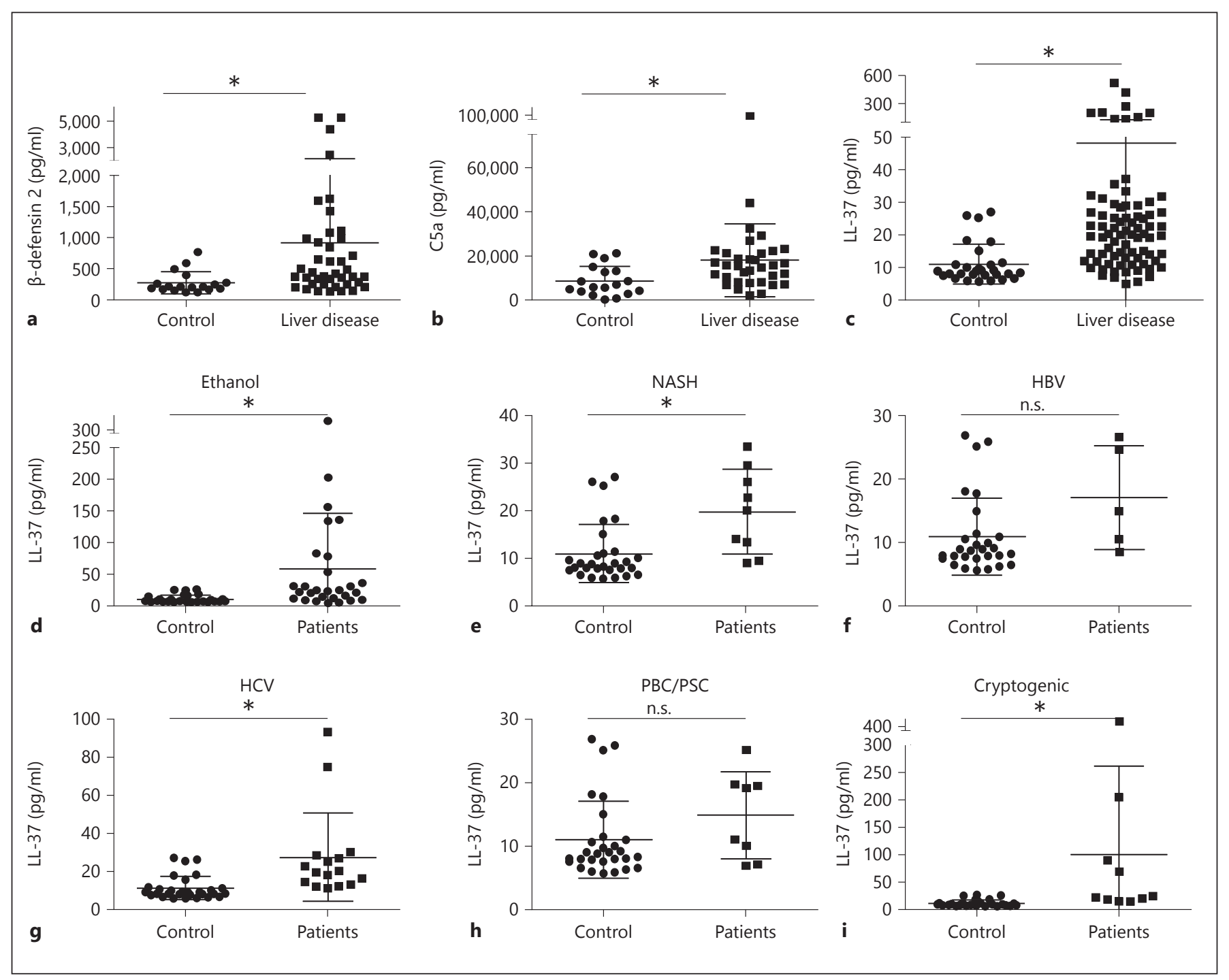

Fig. 1. Serum level of beta-defensin 2, C5a, and LL-37 peptides in human samples. Serum expression levels of antimicrobial peptides beta-defensin 2 (a), C5a (b), and LL-37 (c) were determined by ELISA. Therefore, serum samples of healthy individuals were compared with serum samples of patients with liver disease $(\mathbf{a}, \mathbf{b}-42$ patients). LL-37 serum samples (80 patients) were further grouped

\section{CRAMP-KO Mice Show an Enhanced Liver Fat}

\section{Accumulation after MCD Treatment}

To verify the significance of increased serum level of AMPs (especially of LL-37) and C5a in patients with liver diseases, mice deficient for CRAMP (CRAMP-KO) - the mouse homolog of the human cathelicidin LL-37 - were applied to two different and widely used models of chronic liver injury: methionine/choline-deficient (MCD) diet and bile-duct ligation (BDL). The pathogenesis of both mouse models was shown to be related to gut-derived bac- for different underlying liver disease entities $(\mathbf{d}-\mathbf{i})$. Displayed are the sub-analyses for ethanol-induced injury, NASH, HBC, HCV, cryptogenic liver diseases, and PBC/PSC. Error bars represent mean values with standard deviation. Significances were determined by an unpaired Student's t test $(* \mathrm{p}<0.05)$.

teria mediated by hepatopetal portal blood flow $[14,17,18]$ and can therefore be used as a tool to better understand the functional role of AMPs during liver pathogenesis.

CRAMP-KO mice were first treated with MCD diet for 7 days and 28 days to investigate the impact of LL37/CRAMP on the course of metabolic liver injury. Serum analysis after 7 days displayed a significantly increased ALT level in CRAMP-KO animals (fig. 2a), thus indicating a greater degree of liver injury within this group. Further morphological analysis of those mice re- 


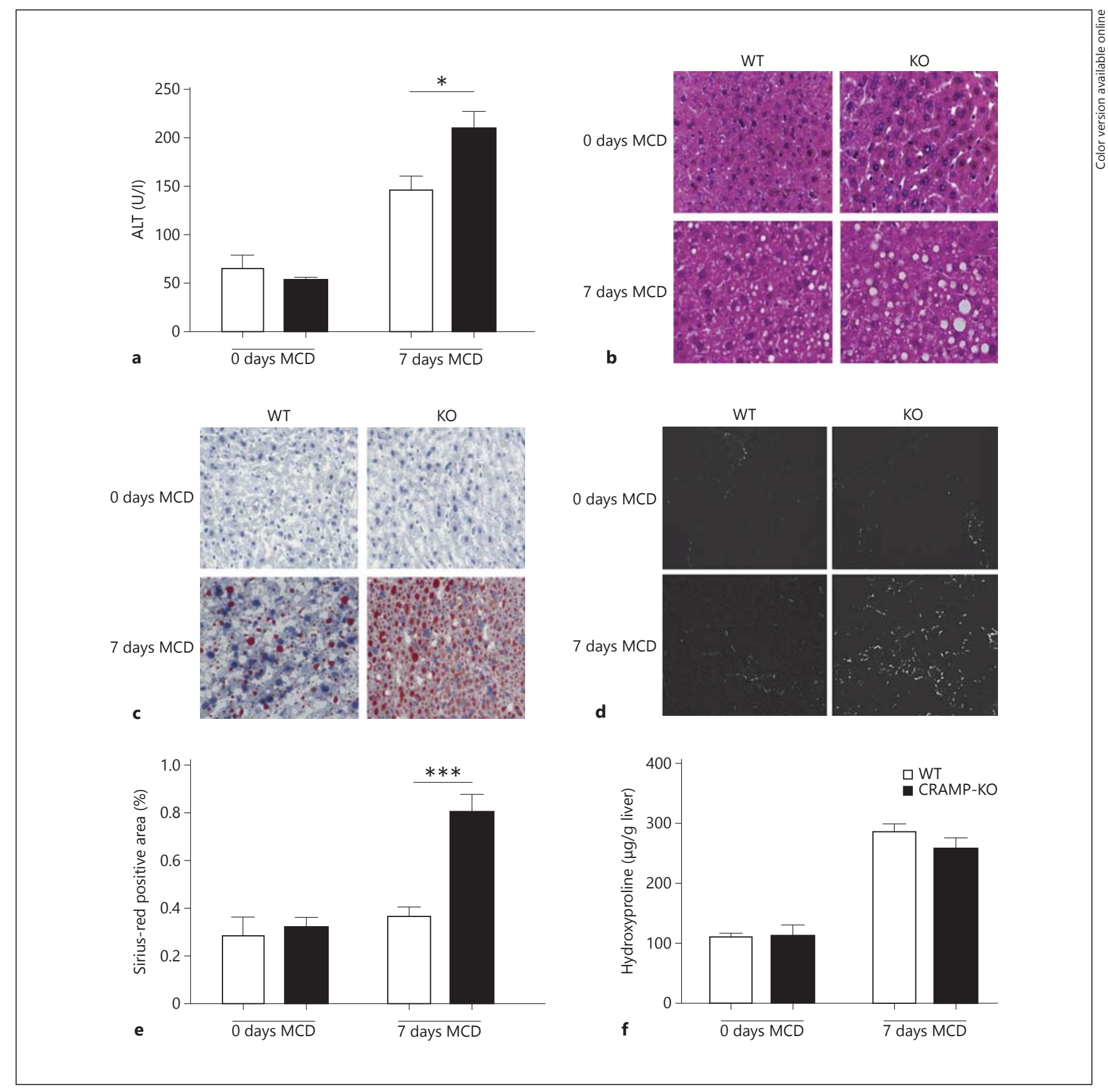

Fig. 2. a Enhanced hepatic fat accumulation after MCD treatment. Displayed are serum alanine aminotransferase (ALT) levels of WT and CRAMP-KO mice $0 \mathrm{~d}$ and after 7 days of feeding a methioninecholine deficient diet (7 days MCD). Values are means \pm SEM. $* \mathrm{p}<0.05)$. b Representative images of H\&E-stained histological slides of WT and CRAMP-KO animals (untreated and MCD fed for 7 days, $40 \times$ magnification). c Displayed are Oil-Red-O -stained liver sections from WT and CRAMP-KO animals (untreated and after 7 days of MCD feeding, 7 days MCD) showing the progressive increase of Oil-Red-O staining in CRAMP-KO mice after MCD feeding ( $40 \times$ magnification). d, e Depicted are representative images and a quantitative analysis of Sirius-red staining showing the beginning of fibrosis in CRAMP-KO animals after 7 days of MCD feeding ( 7 days MCD) in comparison to MCD-fed wild type and to untreated controls (magnification of $\times 40$. ${ }^{* * *} p<0,001$ ). $f$ Displayed are hydroxyproline levels of untreated WT and CRAMPKO mice (0 days MCD) and 7 days after MCD feeding (7 days MCD). 
vealed the appearance of more ballooned hepatocytes and lipid droplets within their livers (data are displayed as $\mathrm{H} \& \mathrm{E}$ and Oil-red-O staining; fig. 2b, c). There was also a tendency toward an earlier and stronger accumulation of extracellular matrix (fig. 2d-f) in CRAMP-KO animals.

Analysis of the $28 \mathrm{~d}$ time point during MCD feeding showed a further progressive accumulation of hepatic fat in CRAMP-KO mice, although the elevated transaminases were not significantly different between subgroups anymore (fig. 3a). However, at this time point, still increasing fatty liver degeneration (fig. 3b) in mice lacking CRAMP could be related to extracellular matrix accumulation in both groups of animals (fig. 3c-e). Those findings were confirmed by subsequently performed Oil-red-O stainings (fig. 3f). Biochemical quantification brought further evidence for a clearly enhanced hepatic triglyceride accumulation (fig. $3 \mathrm{~g}$ ) in CRAMP-KO mice.

\section{BDL Leads to Enhanced Liver Fibrosis Progression in CRAMP-KO Mice}

Next, we applied WT and CRAMP-KO mice to bileduct ligation. This model shows the clearest link to an involvement of intestinal bacterial translocation [14] as a contributor for liver pathogenesis. CRAMP-KO mice displayed enhanced serum ALT level after 7 days of BDL (fig. 4a). Morphometric analysis of livers from bile-ductligated CRAMP-KO mice and their respective controls then unraveled more liver tissue remodeling (fig. 4b). Here, livers of CRAMP-KO mice showed more and larger necrotic areas covering the analyzed liver tissue. Interestingly, we observed a significant enhanced cell proliferation (fig. 4c) within the same group. TUNEL analysis undertaken to detect apoptotic cells within the hepatic tissue, however, did not show differences between both groups (fig. 4d).

Fig. 3. Long-term changes after MCD treatment. a Displayed are serum alanine aminotransferase (ALT) levels of WT and CRAMPKO mice at 0 days and after 28 day of feeding a methionine-choline deficient diet (28 days MCD). b Exhibited are representative images of H\&E-stained histological slides of WT and CRAMP-KO animals ( 0 days MCD control and 28 days MCD) at a magnification of $40 \times$. Treated CRAMP-KO animals show a stronger progressing steatosis in comparison to fed WT mice. c, d Representative images and a quantitative analysis of Sirius-red staining show the enhanced fibrosis progression in CRAMP-KO animals after 28 days of MCD feeding (28 days MCD) in comparison to the fed
To determine whether these early changes would result in long-term differences within the hepatic tissue architecture, WT and CRAMP-KO mice were further analyzed $28 \mathrm{~d}$ after bile-duct ligation. Transaminases were elevated in both groups (fig. 5a). Interestingly, histomorphological analysis then unraveled a significantly stronger liver remodeling and extracellular matrix accumulation in mice lacking CRAMP (fig. 5b). This became evident by more bridging fibrosis and an enhanced Sirius-red staining (fig. 5c). By collagen immunofluorescence and the use of a hydroxyproline assay (fig. 5d, e), we were able to biochemically confirm the increased hepatic collagen content in CRAMP-KO mice.

\section{Discussion}

There is compelling evidence coming from numerous human and rodent animal model studies for the importance of defensins and microbial peptides during the innate phase of immune response. The most evidence for their disease-modifying role comes from studies in human subjects of the respiratory tract, the skin, as well as from those with inflammatory bowel disease and diarrhea. In general, defensins and AMPs such as LL-37 have two major functions in host defence: direct inhibition of pathogens and modulation of other innate and adaptive immune responses [19]. However, data about their involvement during the progression of liver diseases are rare. Our results now show a positive correlation between liver diseases in general and serum levels of hBD2, LL-37, and the complement factor C5a. Interestingly, serum levels of all three parameters were within a narrow range in control patients, but quite differently elevated in patients with liver diseases. Some patients showed only minor changes, whereas others displayed a largely induced level, thus reflecting a broad variability in AMP serum level.

wild-type and to untreated controls ( 0 days MCD) at a magnification of $40 \times$. e Shown are hydroxyproline levels of untreated WT and CRAMP-KO mice ( 0 days MCD) and 28 days after MCD feeding (28 days MCD). $\mathbf{f}$ Microscopy of Oil-red-O stained liver sections from WT and CRAMP-KO animals (untreated 0 day MCD and after 28 days of MCD feeding, 28 days MCD) showing the progressive increase of Oil-red-O staining in WT and CRAMPKO mice after MCD feeding with an even more Oil-red-O positive area in the CRAMP-KO at a (40× magnification). $\mathbf{g}$ Displayed are hepatic triglyceride levels of WT and CRAMP-KO mice 28 days after MCD feeding) ( $\mathrm{p}=0.06)$.

(For figure see next page.) 


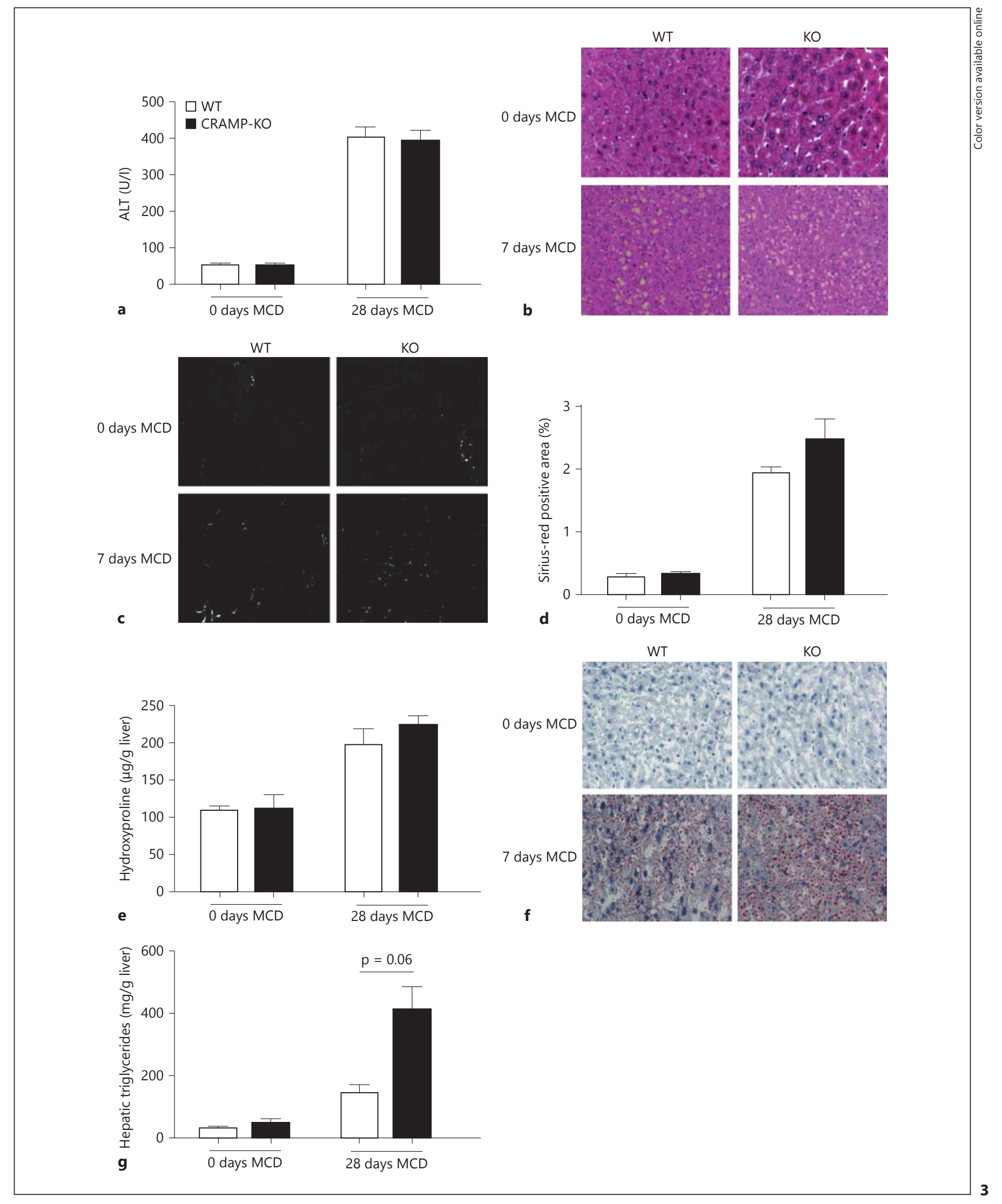




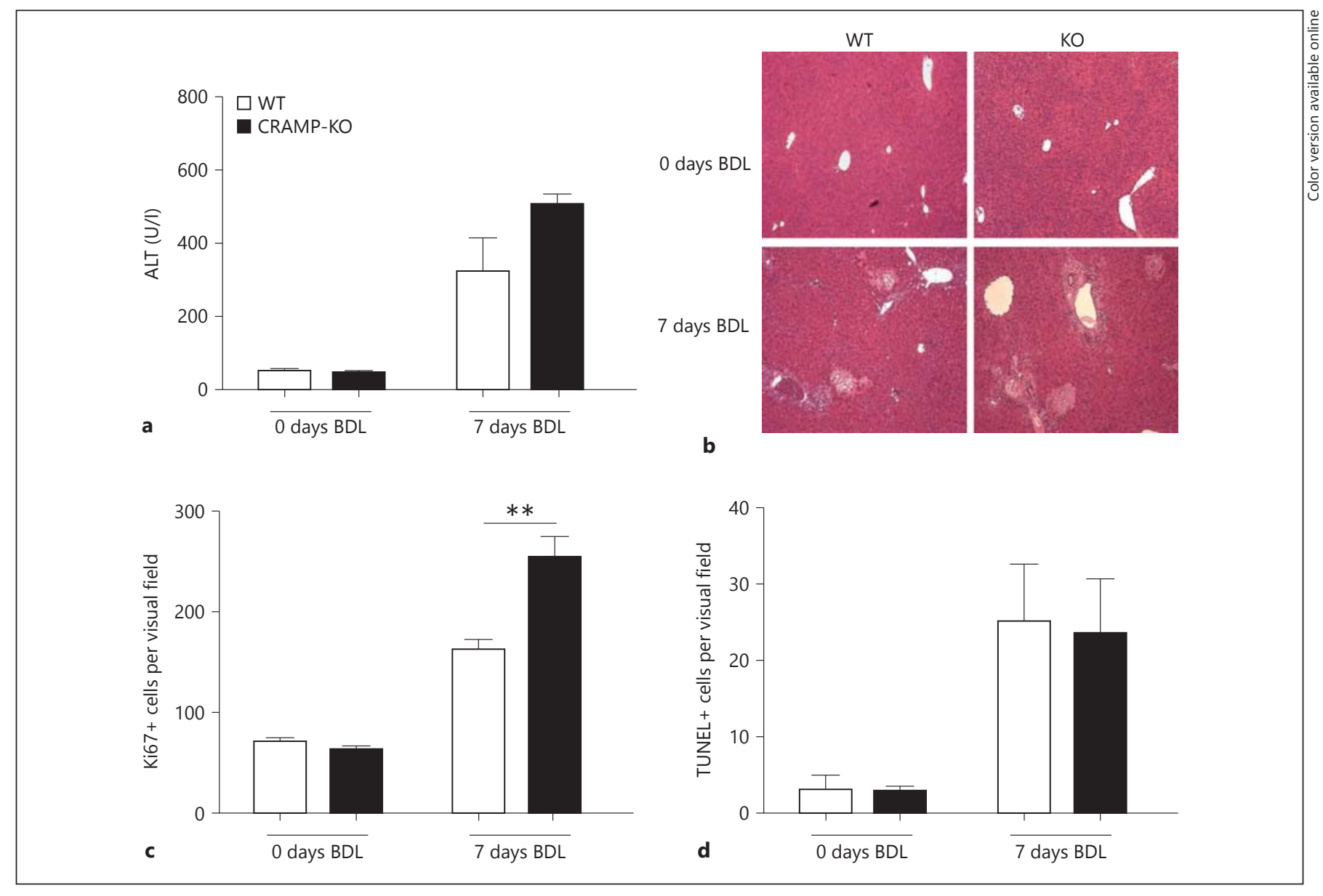

Fig. 4. Inflammation and regeneration of hepatic tissue after BDL, a Displayed are serum alanine aminotransferase (ALT) levels of WT and CRAMP-KO mice untreated ( 0 days BDL) and 7 days after bile-duct ligation (7 days BDL). b Representative images of $\mathrm{H} \& \mathrm{E}$-stained histological slides of WT and CRAMP KO animals (untreated and 7 days after BDL) are shown at a magnification of
$10 \times$. c Presented are Ki67-positive cells per visual field of WT and CRAMP-KO mice (untreated (0 days BDL) and 7 days after bileduct ligation $(7$ days BDL) $(* * \mathrm{p}<0.01))$. d Shown are TUNELpositive cells per visual field of WT and CRAMP-KO mice untreated (0 days BDL) and 7 days after bile-duct ligation (7 days $\mathrm{BDL})$.
Therefore, most liver disease entities displayed a significantly elevated LL-37 serum level, as shown for ethanolinduced injury, NASH, HCV, and cryptogenic liver diseases. HBV and PBC/PSC patients, however, showed a clearly elevated LL-37 level, although not reaching the level of significance. Further clear correlations with disease progression (child status, bilirubin level, etc.) could not be demonstrated (data not shown).

Since CRAMP-KO mice [16] are available, a growing body of data is being generated, which illustrates its function in mouse models of various diseases. Most of the current knowledge comes from lung injury models, where murine CRAMP can act both anti- and proinflammatory
[20]. Thus, its role during the chemotactic recruitment of neutrophils remains two sided. Interestingly, under some conditions - such as self-limiting respiratory diseases large numbers of neutrophils can be recruited, which can release enormous amounts of AMP.

One can imagine that the first, rather proinflammatory step might help at the end to combat the infection. Interestingly, LL-37 can also interfere with apoptosis induction in neutrophils through a formyl peptide receptor-mediated mechanism that was accompanied with the phosphorylation of ERK-1/2, expression of Bcl-x(L) (an antiapoptotic protein), and inhibition of caspase- 3 activity. This suppression of neutrophil apoptosis then results 

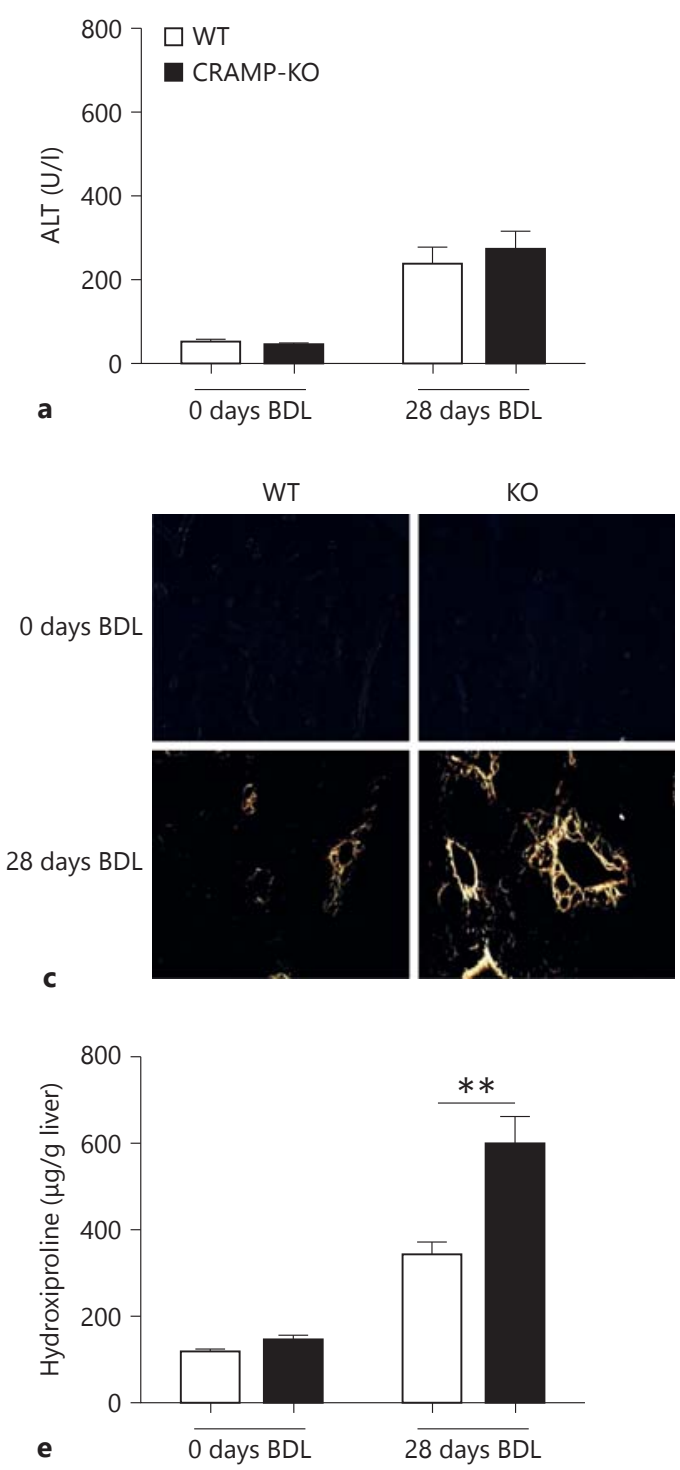
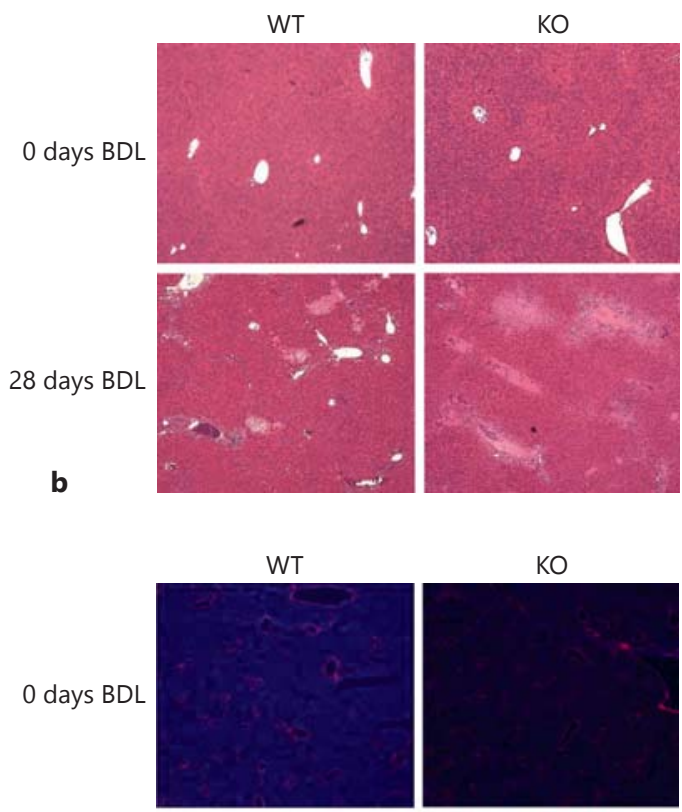

28 days BDL
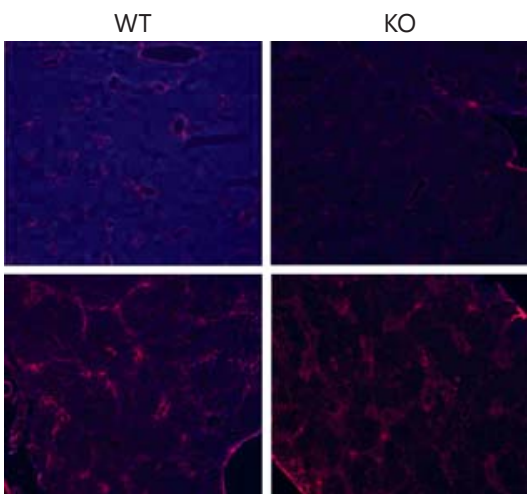

d

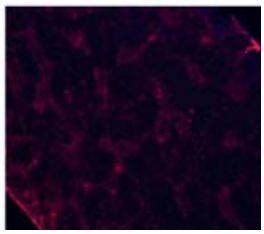

Fig. 5. Long-term changes after BDL. a Displayed are serum alanine aminotransferase (ALT) levels of WT and CRAMP-KO mice untreated ( 0 days BDL) and 28 days after bile-duct ligation (28 days BDL). b Representative images of H\&E-stained histological slides of WT and CRAMP-KO animals (untreated and 7 days after $\mathrm{BDL}$ ) are shown at a magnification of $10 \times$. Livers of CRAMP-KO mice show more liver damage (necrosis and bile-duct infarcts) over time. c Representative images of Sirius-red staining display an enhanced fibrosis progression in CRAMP-KO animals after 28 days of BDL (28 days BDL) in comparison to bile-duct-ligated WT mice and to untreated controls ( 0 days BDL) at a magnification of $5 \times$. d Depicted are images of collagen immunohistochemical staining at a magnification of $5 \mathrm{x}$, indicating that fibrosis in CRAMP-KO mice at 28 days after BDL is increased compared with WT mice at 28 days after BDL and untreated controls (0 days BDL). e Shown are hydroxyproline levels of WT and CRAMP-KO mice untreated $(0$ days BDL) and 28 days after BDL $(28$ days BDL) $(* * \mathrm{p}<0.01)$. 
in the prolongation of their lifespan and may be advantageous for host defense against bacterial invasion [21].

Our subsequently performed animal experiments showed that the lack of CRAMP in mice renders them, in general, more susceptible for liver injury. Although we used a metabolic and a cholestatic model, the observed liver injury patterns in CRAMP-KO mice were similar. Within the MCD model, the predominant phenotype was fatty degeneration, whereas BDL provoked more matrix accumulation, resulting in a stronger onset of liver fibrosis if CRAMP was absent. Cell-protective effects of LL-37/ CRAMP have long been described [22]. Potential underlying mechanisms were mostly characterized with regard to pathogen clearance on a cellular level using tissue culture experiments. Observed effects in hepatic injury models, however, can be better explained by more indirect, anti-inflammatory functions of the peptide. Of special interest here is the interference of LL-37 with the LPS-signaling pathway. In epithelial cell lines, it was shown that LPS/cyclohexemide-induced cell death could be inhibited through LL-37 stimulation. This interaction most likely happens at the level of the CD14/TLR4 interaction, although questions still remain here. Through this inhibition, TLR4-induced JNK activation and apoptotic signaling can be inhibited [23]. An interaction with the TLR4 pathways becomes of special interest, when one takes into account that mice deficient in TLR4 signaling are protected against BDL [14] or MCD-induced liver injury [18]. Under both conditions in TLR4-KO mice as well as after gut sterilization, liver fibrosis development was largely attenuated.

Several mouse homologs for human beta defensins, another class of AMPs involved in the regulation of the innate immune response, have been found. However, the general role of defensins and other AMP homologs in mice is not fully understood. Human-beta defensine-1 KO mice showed a slower clearance of bacterial species during lung injury but no obvious phenotype with regard to their inflammatory response [24]. Thus, one has to consider that different defensins might rather act cooperatively together and that therefore the deletion of individual members is rather uncritical, as one might be able to compensate for another. Especially human beta defensins 2-4 are expressed during infectious or inflammatory stimuli and thus serve as important co-defenders against microbial components and immune regulators. Our findings can complete earlier data on $\mathrm{hBD} 2$ expression during cholangitis [25], where it was shown that especially hBD2 was expressed in cells of the biliary epithelium. However, it remains elusive as to whether our correlation of hBD2 expression in sera of patients with hepatic diseases might reflect an increased remodeling of biliary epithelia, which occurs during the development of liver diseases. Complement $5 \mathrm{a}$ (C5a), which is also known as a critical modulator of liver immunity, was found to be increased in patients with liver diseases as well. It was previously shown that it was increased in hepatitis B patients [26]. The authors showed that C5a could activate hepatic stellate cells and stimulate matrix production. Another work by Hillebrandt et al. [27] identified C5a as an important quantitative trait gene that was associated with advanced liver fibrosis.

Our study now shows for the first time a positive correlation of antimicrobial peptide serum levels with the presence of liver disease. This became true for different liver disease entities, as from viral hepatitis $(\mathrm{HCV})$ to NASH clearly enhanced LL-37 could be detected. We therefore think that the release of AMPs during liver disease represents a rather general and not a disease-specific mechanism. To determine whether this finding reflects a reaction against enhanced bacterial translocation in the gut or an unspecific inflammatory response remains to be determined through future investigations. Mouse animal data further suggest a protective role of LL-37/CRAMP, particular during hepatic fatty liver degeneration and/or fibrosis development in mice (bile-duct ligation model), both of which are conditions that also develop in humans as a general response to chronic hepatic injury. However, further research is needed to fully understand the exact role of LL-37/CRAMP and other individual AMPs in innate immune response during the pathogenesis of liver diseases. This will help to better explore their potential for prognostic or even therapeutic uses.

\section{Funding}

Konrad Streetz and Daniela Kroy were supported by a grant of the Deutsche Forschungsgemeinschaft (DFG), grant SFB/TRR57, P22.

Lars-Ove Brandenburg was supported by the Else Kröner (with Umlaut Ö) Fresenius-Stiftung.

\section{Disclosure Statement}

The authors declare that they have no competing financial interests. 


\section{References}

1 Brandenburg L-O, Merres J, Albrecht L-J, Varoga $\mathrm{D}$, Pufe T: Antimicrobial peptides: multifunctional drugs for different applications. Polymers 2012;4:539-560.

2 Zanetti M: The role of cathelicidins in the innate host defenses of mammals. Curr Issues Mol Biol 2005;7:179-196.

3 van der Does AM, Beekhuizen H, Ravensbergen B, Vos T, Ottenhoff TH, van Dissel JT, Drijfhout JW, Hiemstra PS, Nibbering PH: LL-37 directs macrophage differentiation toward macrophages with a proinflammatory signature. J Immunol 2010;185:1442-1449.

4 Rosenfeld Y, Papo N, Shai Y: Endotoxin (lipopolysaccharide) neutralization by innate immunity host-defense peptides. Peptide properties and plausible modes of action. J Biol Chem 2006;281:1636-1643.

5 Brown KL, Poon GF, Birkenhead D, Pena OM, Falsafi R, Dahlgren C, Karlsson A, Bylund J, Hancock RE, Johnson P: Host defense peptide LL-37 selectively reduces proinflammatory macrophage responses. J Immunol 2011;186:5497-5505.

6 Bucki R, Leszczyńska K, Namiot A, Sokołowski W: Cathelicidin LL-37: a multitask antimicrobial peptide. Arch Immunol Ther Exp (Warsz) 2010;58:15-25.

7 Gombart AF, Borregaard N, Koeffler HP: Human cathelicidin antimicrobial peptide (CAMP) gene is a direct target of the vitamin $\mathrm{D}$ receptor and is strongly up-regulated in myeloid cells by 1,25-dihydroxyvitamin D3. FASEB J 2005;19:1067-1077.

8 Park K, Elias PM, Oda Y, Mackenzie D, Mauro T, Holleran WM, Uchida Y: Regulation of cathelicidin antimicrobial peptide expression by an endoplasmic reticulum (ER) stress signaling, vitamin d receptor-independent pathway. J Biol Chem 2011;286:34121-34130.

9 Bergman P, Termen S, Johansson L, Nystrom L, Arenas E, Jonsson AB, Hokfelt T, Gudmundsson GH, Agerberth B: The antimicrobial peptide rcramp is present in the central nervous system of the rat. J Neurochem 2005; 93:1132-1140.
10 Chromek M, Slamova Z, Bergman P, Kovacs L, Podracka L, Ehren I, Hokfelt T, Gudmundsson GH, Gallo RL, Agerberth B, Brauner A: The antimicrobial peptide cathelicidin protects the urinary tract against invasive bacterial infection. Nat Med 2006;12:636-641.

11 Brandenburg LO, Varoga D, Nicolaeva N, Leib SL, Wilms H, Podschun R, Wruck CJ, Schroder JM, Pufe T, Lucius R: Role of glial cells in the functional expression of LL-37/rat cathelin-related antimicrobial peptide in meningitis. J Neuropathol Exp Neurol 2008; 67:1041-1054.

12 Merres J, Höss J, Albrecht LJ, Kress E, Soehnlein O, Jansen S, Pufe T, Tauber SC, Brandenburg LO: Role of the cathelicidin-related antimicrobial peptide in inflammation and mortality in a mouse model of bacterial meningitis. J Innate Immun 2014;6:205-218.

13 Kesar V, Odin JA: Toll-like receptors and liver disease. Liver Int 2014;34:184-196.

14 Seki E, De Minicis S, Osterreicher CH, Kluwe J, Osawa Y, Brenner DA, Schwabe RF: TLR4 enhances TGF-beta signaling and hepatic fibrosis. Nat Med 2007;13:1324-1332.

15 Giebeler A, Brandenburg LO, Kaldenbach M, Erschfeld S, Wasmuth H, Wruck C, Trautwein C, Streetz KL: Lack of hepatic c-Met and gp130 expression is associated with an impaired antibacterial response and higher lethality after bile duct ligation. Lab Invest 2012;92:1726-1737.

16 Nizet V, Ohtake T, Lauth X, Trowbridge J, Rudisill J, Dorschner RA, Pestonjamasp V, Piraino J, Huttner K, Gallo RL: Innate antimicrobial peptide protects the skin from invasive bacterial infection. Nature 2001;414:454-457.

17 Kudo H, Takahara T, Yata Y, Kawai K, Zhang W, Sugiyama T: Lipopolysaccharide triggered TNF-alpha-induced hepatocyte apoptosis in a murine non-alcoholic steatohepatitis model. J Hepatol 2009;51:168-175.

18 Csak T, Velayudham A, Hritz I, Petrasek J, Levin I, Lippai D, Catalano D, Mandrekar P, Dolganiuc A, Kurt-Jones E, Szabo G: Deficiency in myeloid differentiation factor- 2 and toll-like receptor 4 expression attenuates nonalcoholic steatohepatitis and fibrosis in mice. Am J Physiol Gastrointest Liver Physiol 2011; 300:G433-G441.
19 Auvynet C, Rosenstein Y: Multifunctional host defense peptides: antimicrobial peptides, the small yet big players in innate and adaptive immunity. Febs J 2009;276:6497-6508.

20 Kovach MA, Ballinger MN, Newstead MW, Zeng X, Bhan U, Yu FS, Moore BB, Gallo RL, Standiford TJ: Cathelicidin-related antimicrobial peptide is required for effective lung mucosal immunity in Gram-negative bacterial pneumonia. J Immunol 2012;189:304-311.

21 Nagaoka I, Tamura H, Hirata M: An antimicrobial cathelicidin peptide, human CAP18/ LL-37, suppresses neutrophil apoptosis via the activation of formyl-peptide receptorlike 1 and P2X7. J Immunol 2006;176:30443052.

22 van der Does AM, Bergman P, Agerberth B, Lindbom L: Induction of the human cathelicidin LL-37 as a novel treatment against bacterial infections. J Leukoc Biol 2012;92:735742 .

23 Suzuki K, Murakami T, Kuwahara-Arai K, Tamura H, Hiramatsu K, Nagaoka I: Human anti-microbial cathelicidin peptide LL-37 suppresses the LPS-induced apoptosis of endothelial cells. Int Immunol 2011;23:185193.

24 Moser C, Weiner DJ, Lysenko E, Bals R, Weiser JN, Wilson JM: Beta-defensin 1 contributes to pulmonary innate immunity in mice. Infect Immun 2002;70:3068-3072.

25 Harada K, Ohba K, Ozaki S, Isse K, Hirayama T, Wada A, Nakanuma Y: Peptide antibiotic human beta-defensin-1 and -2 contribute to antimicrobial defense of the intrahepatic biliary tree. Hepatology 2004;40:925-932.

26 Xu R, Lin F, He J, Jin L, Zhang JY, Fu J, Liu H, Wang S, Zhang Z, Wang FS: Complement $5 \mathrm{a}$ stimulates hepatic stellate cells in vitro, and is increased in the plasma of patients with chronic hepatitis B. Immunology 2013;138: 228-234.

27 Hillebrandt S, Wasmuth HE, Weiskirchen R, Hellerbrand C, Keppeler H, Werth A, Schirin-Sokhan R, Wilkens G, Geier A, Lorenzen J, Kohl J, Gressner AM, Matern S, Lammert F: Complement factor 5 is a quantitative trait gene that modifies liver fibrogenesis in mice and humans. Nat Genet 2005;37:835-843. 